\title{
Direct Numerical Simulation of Fracture Behaviour for Random Short Wood Fibres Reinforced Composites, Comparison with Digital Image Correlation Experiments
}

\author{
Min SHEN ${ }^{1,2, a}$, Fabienne TOUCHARD ${ }^{1, b}$, Gérard BEZINE ${ }^{1}$, Jean BRILLAUD ${ }^{1}$ \\ ${ }^{1}$ Laboratoire de Mécanique et de Physique des Matériaux, UMR CNRS 6617, ENSMA,1 Av \\ Clément Ader, BP 40109 , 86961 Futuroscope-Chasseneuil du Poitou FRANCE \\ ${ }^{2}$ Department of Mechanics, Tianjin University, Tianjin 300072, PR China
}

\begin{abstract}
The work is to predict fracture behaviour of bio-composites from the tensile properties of its components. In this work, we have realized a direct numerical simulation of fracture behaviour for random short spruce fibers reinforced composites. For calculations, wood fibers have been considered as linear elastic bodies, polypropylene matrix as an elastic-plastic material. Then, numerical results have been compared with experimental results that have been obtained by digital image correlation. This comparison indicates that random fiber FE model of random short spruce fibers reinforced composites can be able to fairly reflect the influence of random fibers microstructure in the composite on its fracture behavior. The calculation of both random fiber and homogeneous FE model and their comparison with experiments show that the average values of J-integral in a region in the front of the crack tip from both numerical FE models are in good agreement with the average $\mathrm{J}$ value of DIC experiment in the same region when the numerical and experimental CT specimens of the short spruce fiber reinforced composite are subjected to the same extension at their loading point.
\end{abstract}

\section{Introduction}

The random spruce short fibre reinforced polypropylene (PP) composite is one kind of biocomposites with higher fibre volume content. This kind biocomposite is gradually used as the preferred material in the design of inner components or trim panels in vehicles such as automobile and bullet train, because of its lightweight and high fracture toughness. With increasing the awareness of environmental protection and the reuse of natural resources, the researches of the bio composite material are attracting more and more attention because it is fully biodegradable, recyclable and environmentally-friendly, meanwhile it is widely used in large amount in the automotive industry which has high updated rate of the annual automotive components [1].

Polypropylene (PP) is used for a large number of applications because it is cheap, lightweight, easily recyclable and modifiable to achieve specific requirements [1,2]. In order to be able to meet the high demands on stiffness and strength, polypropylene must often be reinforced. A variety of

\footnotetext{
a e-mail: minshen@tju.edu.cn

be-mail: fabienne.touchard@1mpm.ensma.fr
} 
natural fibres are available for reinforcement in polypropylene, for example: flax, hemp, sisal, bamboo [3]. In the present study, spruce fibres (sawdust) have been incorporated successfully into polypropylene with different fibre weight fractions in order to produce the spruce fibres/PP composite [4].

However, in spite of the superior properties of the composite materials, the use of composite materials is still limited. One of the main reasons for this limited application is the difficulty in reliable prediction of the mechanical behavior of composite materials. A large amount of research about these aspects has been published $[5,6]$. However, in contrast to fracture behavior of metallic components as represented by plasticity phenomena, the understanding of the corresponding behavior in short spruces fiber/PP composites is virtually nonexistent. The failure and fracture of these polymeric structures are correspondingly poorly understood.

The fracture mechanism of polypropylene under static loading condition has been studied by three- and four-point bending tests [7], and the complexity of the crack tip damage zone is shown by means of SEM and optical microscope analysis. The non-local characteristics of short glass-fibrereinforced polypropylene are studied by a mixed numerical-experimental approach of the gradientenhanced damage model and the DIC experiments [8]. The fracture behavior of a short fiber composite biomaterial is predicted from its tensile properties by using nonlinear finite element method and its fracture testing [9].

The traditional optical measurements [10], such as photoelasticity, moire, holography, speckle interferometrgy, shearography, etc., which need to pretreat the surface of object with spraying paint, coating and reproducing grid, and even more to make model, may give full-field information of object deformation from the interferometric fringe. Such techniques suffer from characteristic limitations and are commonly performed in the laboratory. The digital image correlation method (DICM) is based on the correlation of grey values of successive digital images of the undeformed and the deformed specimen $[11,12]$ It can directly measure the transformation by tracking the gray value pattern in small local neighborhoods commonly referred to as subsets.

A technique of measuring the whole-field of strain components on the object surface is established in LMPM by means of combining DIC method with the ABAQUS finite element (FE) code [13]. A GRANU.EXE program of DICM has been developed in order to directly measure the displacements on the object surface. Then the strain field is obtained by using FE software to calculate the spatial derivative of the displacement field. Each measurement point becomes a node of the finite-element mesh and the measured displacement vectors are imposed as boundary conditions.

Using DICM, the fracture and tensile behavior of short spruces fiber/PP composite have been studied by F. Touchard and T.Q. Lam et al. in LMPM $[14,15]$. For CT specimen of the composite, the value of J-integral and the stress field are obtained by using their DICM with a suitable material law. Their experimental results of the composite show the influence of the microstructure of fibers in the material on its fracture behavior.

For the design and application of this natural spruce fibres reinforced PP polymer composite, it is still a new and very important subject to study the influence of the natural fibre microstructure on fracture and tensile behaviour of this material.

In this work, a method based on coupling between direct numerical simulation and DICM is used to study the influence of the random spruce fibre microstructure on fracture behaviour and parameters of the spruce fibre/PP biocomposite. The numerical results will be compared with above DIC experimental results. This comparisons between experimental and numerical results for the biocomposite will help ones to detail understand the results and phenomenon of DIC experiments; to improve the numerical models in order to be more close to the experimental phenomenon and to increase the knowledge about the fracture behavior of this bio-composite.

\section{Used materials and Specimen}

The studied materials are polypropylene (PP) and spruce fibres coming from wood industrial wastes, with length and diameter about respectively $800 \mu \mathrm{m}$ and $350 \mu \mathrm{m}$. The used polypropylene (PP) is the 
APPRYL 3400 MA1 supplied by ATOFINA. The proportion of fibre weight is $30 \%$. The tensile properties of pure polypropylene (PP) and spruce fiber are given in the Table 1 .

Table 1. Tensile properties of materials at $10 \mathrm{~mm} / \mathrm{min}$

\begin{tabular}{|c|c|c|c|c|c|}
\hline Materials & $\begin{array}{c}\text { Young's modulus } \\
\mathrm{E}(\mathrm{MPa})\end{array}$ & $\begin{array}{c}\text { Poisson's ratio } \\
\mathbf{v}\end{array}$ & $\begin{array}{c}\text { Yield strength } \\
\sigma_{y}(\mathrm{MPa})\end{array}$ & $\begin{array}{c}\text { Failure strength } \\
\sigma_{f}(\mathrm{MPa})\end{array}$ & $\begin{array}{c}\text { Failure strain } \\
\varepsilon_{f}(\%)\end{array}$ \\
\hline Pure PP & 1573.0 & 0.4 & 41.5 & 49.5 & $88.5 \pm 31.5$ \\
\hline Spruce fiber & 10500.0 & 0.3 & & & \\
\hline
\end{tabular}

Tensile specimens were cut out from $4 \mathrm{~mm}$ thick-panels. Compact Tension (CT) specimens for fracture tests were cut out from $8 \mathrm{~mm}$ thick-panels. The initial length of the pre-crack was about $400 \mu \mathrm{m}$ depending on the specimen, as shown in Fig. 1.

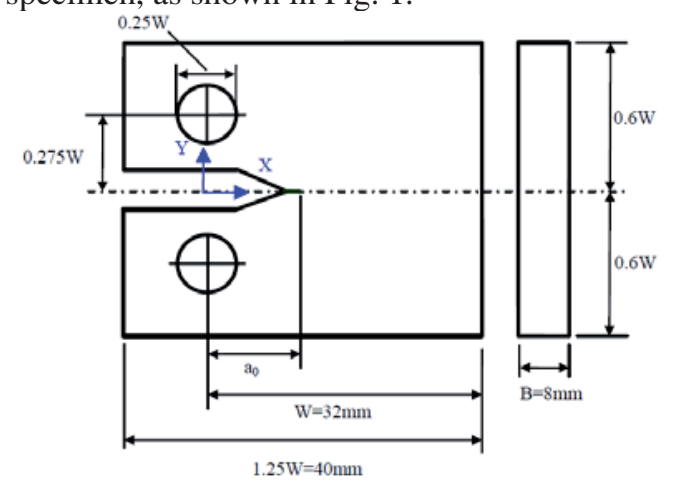

Fig. 1. Compact tension specimen configurations $\left(a_{0}=14.5 \mathrm{~mm}\right.$, as in the document E399[5])

\section{Direct Numerical Simulation of fracture and tensile behaviour}

A finite element model with random spruce fibres for PP composite is developed using ABAQUS code and the pre-processed program of the random fibre elements generation in FORTRAN.

Assumptions adopted for numerical computations: 1.Spruce short-fiber material is elastic, polypropylene matrix (PP) is elastic-plastic material. There is a perfect bond between them at the fiber extremities; 2. There is no stress (or strain) field interaction between spruce short-fibres; 3 . Each fiber segment is embedded in a homogeneous matrix PP medium. Because the Fiber Young's modulus is much higher than the Matrix Young's modulus, we only considered that tensile and compressive deformations are possible, without any bending deformation of the fibres. The spruce fibre is simulated by elastic truss element, and PP matrix is simulated by 2-D solid element, such as T2D2 and CPS8R respectively.

For tensile specimen, the size and geometry of finite element model with random spruce fibers (49.02\% in volume) are given in Fig. 2 in present analysis. The boundary conditions are as follows:

$$
\begin{aligned}
& u(-4, y)=0, y \in[-2.5,2.5] \\
& u(4, y)=u_{0}, y \in[-2.5,2.5] \\
& v(-4,0)=0
\end{aligned}
$$

For CT specimen, the size and geometry of finite element model are given in Fig.3. When it is loaded with a force $\mathrm{F}$ in the $\mathrm{y}$-axis, the boundary conditions are as follows: 


$$
\begin{aligned}
& v(x, 0)=0, \quad x \in(14.5,32] ; \\
& u(14.5,0)=0 \\
& \bar{F}(0,8.8)=F
\end{aligned}
$$

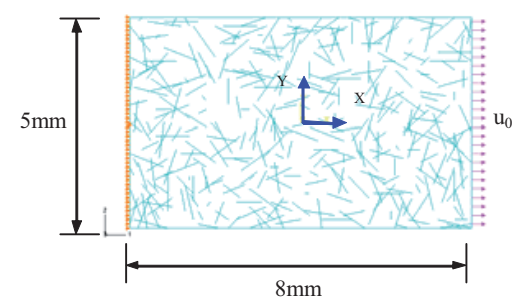

Fig. 2. The finite element model of tensile composite specimen (thickness $2 \mathrm{~mm}$ )

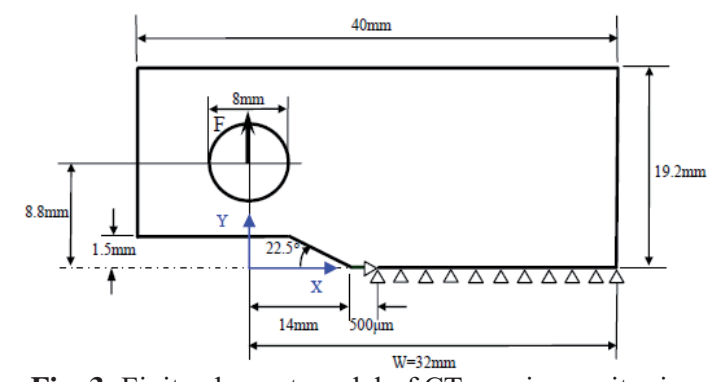

Fig. 3. Finite element model of CT specimen : its size, coordinates and boundary conditions

Direct numerical simulations of fracture and tensile behaviour for random short spruces fibers reinforced composites are carried out.

\section{Comparisons with DICM Experiments}

\subsection{Tension behaviour of spruce fibre/PP composites}

The influence of the fibre content on the tensile behaviour is considered using this FE model with random fibre elements. In Fig.4, the solid lines are experimental stress-strain curves for spruce fibres reinforced PP materials at a constant crosshead displacement rate of $20 \mathrm{~mm} / \mathrm{min}$ at room temperature, the fine dots lines are numerical prediction of the composite FE model. For each fibre volume contents, the comparison of numerical and experimental tensile curves is given in Fig.4. These comparison shows that the numerical tensile curve with lower fibre volume content is closer to experimental one than that with higher fibre volume content.

When fibre diameter is $0.35 \mathrm{~mm}$ and matrix Young's modulus is $1880 \mathrm{MPa}$ at loading rate $20 \mathrm{~mm} / \mathrm{min}$, the numerical true stress-strain curve is agree with experimental for the content of fibre volume 49\%, as shown in Fig.5. In the next section, this numerical stress-strain curve predicted by random fiber FE model is directly used in the homogeneous finite element model of the spruces fiber/PP composite in order to simulate its fracture behaviour.

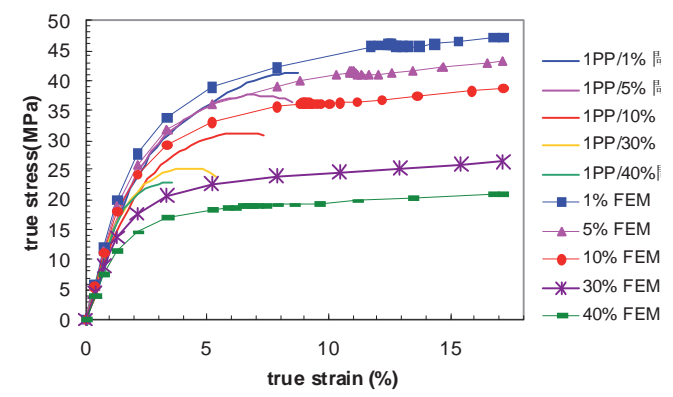

Fig. 4. Experimental and numerical tensile stressstrain curves $(\mathrm{D}=0.6 \mathrm{~mm})$

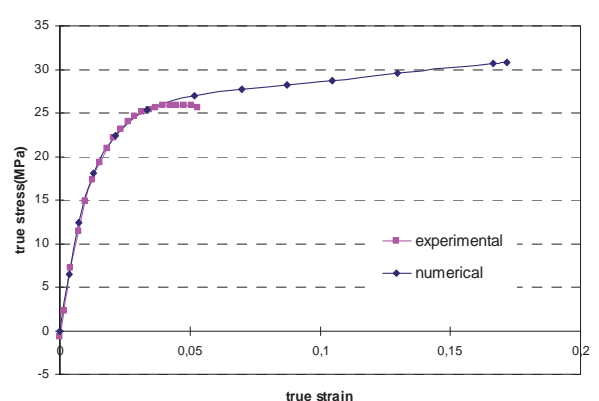

Fig. 5. Experimental and numerical homogeneous true stress vs. strain curves 
To sum up, using the present random fiber finite element model, the tensile simulation shows that the volume content and the diameter of fiber have an effect on the numerical prediction of the tensile behaviour of this short fiber composite. The tensile numerical stress-strain curve which is close to the experimental curve can be predicated by using suitable fiber diameter in the random fiber FE model.

\subsection{Analysis and comparisons of fracture behaviour for $49 \%$ spruce fibre/PP}

The DIC experiments of the spruces fiber/PP composite is the basic of the numerical simulations of both random fiber model and homogeneous model in the present work. The comparison between experiments and both FE models for results of this bio-composite will help ones to detail understand the results and phenomenon of DIC experiments; to improve the numerical models in order to be closer to the experimental phenomenon and to increase the knowledge about the fracture behavior of this bio-composite. It is very important that the numerical results are compared with DIC experiments in this work.

Methods of the comparison numerical simulations with DIC experiments are given in this section. The field of strain of CT composite is obtained from the field of experimental displacements by combining the digital image correlation method (DICM) with ABAQUS code. Then, the field of stresses and the value of J-integral are obtained farther by means of using the experimental stressstrain curve of the bio-composite in ABAQUS code. Using this method, all of the experimental parameters about the fracture behavior of the composite, including the stresses, strains, COD, CTOD and $\mathrm{J}$ integral, are measured directly and indirectly. The results of this way are given by red lines in below Figures.

The random elements of spruces fibers are generated by the FORTRAN preprocessed which is developed in present work. The finite element model of spruce fiber/PP composite with random fibers is created. The fracture behavior and tensile behavior of the bio-composite are directly simulated by using this FE model. The numerical stresses, strains, COD, CTOD and J integral are calculated by the random fiber FE model. The results of this second way are shown by blue lines in following figures.

The third way is the simulation of homogeneous finite element model, its results are shown by green lines in figures. The numerical stress-strain curve predicted by random fiber FE model is directly used in the homogeneous finite element model of the spruces fiber/PP composite in order to simulate its fracture behavior, as above 4.1 section.

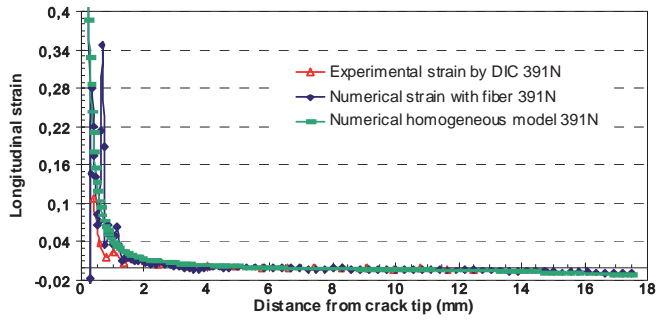

Fig. 6. The $\varepsilon_{y y}$ in net section at load $391 \mathrm{~N}$ for composite with $49 \%$ fiber in volume using DIC experimental and numerical random and homogeneous model

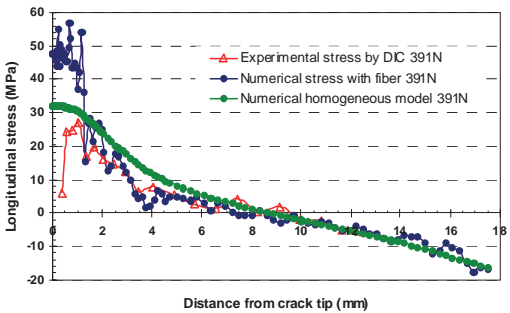

Fig. 7. The $\sigma_{y y}$ in net section at load $391 \mathrm{~N}$ for composite with $49 \%$ fiber in volume using DIC experimental and numerical random and homogeneous model

Figs. 6 and 7 shows the longitudinal strain $\varepsilon_{\text {yy }}$ and stress $\sigma_{\text {yy }}$ near the crack tip in net section at load $391 \mathrm{~N}$ respectively for composite with $49 \%$ fiber in volume using DIC experimental and numerical random and homogeneous model. From above results, the distributions of strain $\varepsilon_{\mathrm{yy}}$ and stress $\sigma_{\text {yy }}$ of random fiber model reflect the influence of the microstructure of spruces fibers/PP composite on the stress and strain. The comparison between the random and homogeneous models 
shows that the mesoscopic stress and strain of random model are larger than that of the homogeneous model.

The curves of applied load versus extension of experiment and both numerical models are given in Fig. 8 for the composite with $49 \%$ fiber in volume using DIC experimental and numerical random and homogeneous mode. The results show that experimental and both numerical random and homogenous models have different extensions at same applied loads which is higher than $100 \mathrm{~N}$.

Fig. 8 gives the curves of extension versus the average $\mathrm{J}$ value in the above region of the contour position for experiments and both numerical models. It shows that the curves of extension versus the average $\mathbf{J}$ value for different $\mathrm{FE}$ model and experiment are almost same, and both numerical average $\mathbf{J}$ values are very agree with the experimental at same extension load. If the curves of applied load and average $\mathrm{J}$ value versus extension are plotted in the same Fig.8, it is not difficult to explain the difference of average $\mathrm{J}$ values between experiment and both numerical random and homogeneous models at same applied load.

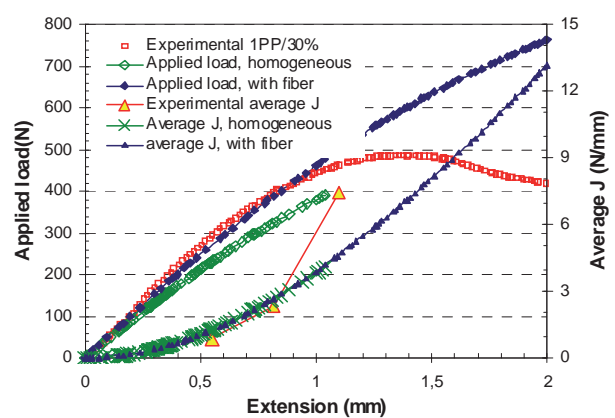

Fig. 8. Average J value and applied load vs. extension for composite with $49 \%$ fiber in volume

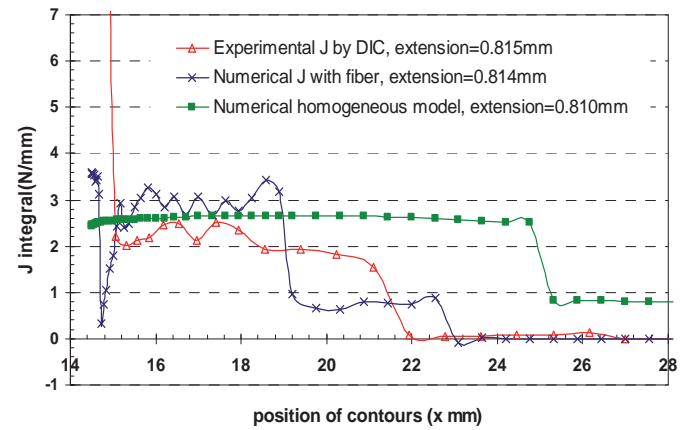

Fig. 9. J integral at $0.81 \mathrm{~mm}$ extension for composite with $49 \%$ fibre in volume using DIC experimental and numerical random and homogeneous model

According to above analysis, if the comparison of the average $\mathbf{J}$ value between experiment and both numerical models is carried out from the point of view of the extension load, their values or curves will be closer at same extension load than at same applied load. Fig.9 gives the new results of the comparison between the experiments and both models at $0.81 \mathrm{~mm}$ extension load. From the view of the extension load, the results of experiment and both random and homogeneous models can be understood deeply.

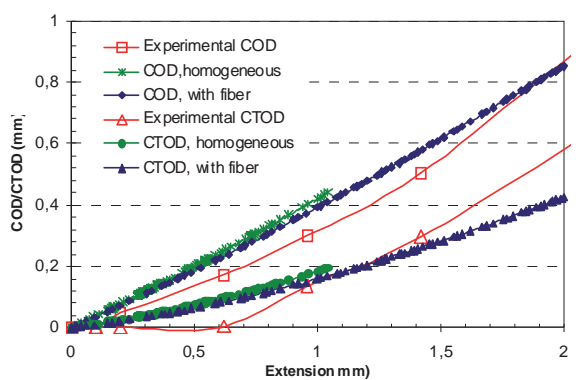

Fig.10. COD and CTOD vs. extension for composite with $49 \%$ fibre in volume

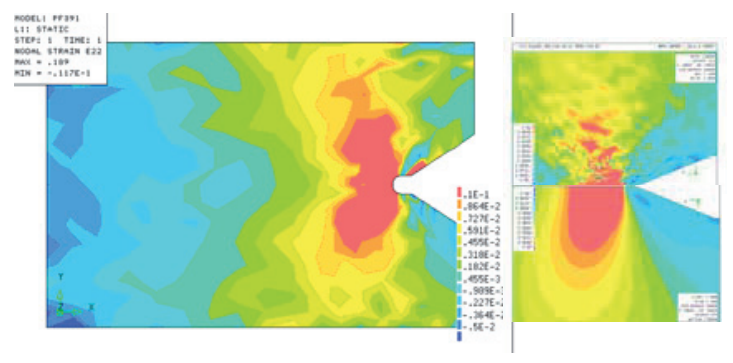

Fig. 11. longitudinal Eyy strain maps in front of the crack tip at applied load $391 \mathrm{~N}$ for spruce fibre/PP composite by DICM, homogeneous model and random fibre model

The curves of COD and CTOD versus extension for composite with $49 \%$ fiber in volume are given in Fig.10 by using DIC experiment and numerical random and homogeneous models. The results show that both numerical curves of COD and CTOD are consistent variety with the 
experimental curves at the beginning. But the prediction of CTOD from both models has difference with experimental CTOD. Due to the simulation of both random fiber and homogeneous models is only suitable for the short fiber reinforce composite without damage, such as fiber-matrix interface debondings and crack growing. The critical values of COD and CTOD should be determined by the further experiments of the composite in order to exactly give the applicable region of the random fiber finite element model.

The longitudinal $\varepsilon_{\text {yy }}$ strain maps in front of the crack tip at applied load $391 \mathrm{~N}$ for spruce fiber/PP composite by DIC method, homogeneous model and random fiber model are given in Fig.11. It can be found from these strain maps that the strain contour of the homogeneous model is smoother than others, and the contour of random fiber model is sharp and fragmentized because of the influence of the microstructure of spruces fibers in the composite. Because the deformation measured by DICM is the average deformation of the region of the correlation sub-window at the measurement point. The pattern of strain contour of DIC experiment is between both numerical ones, not more sharp and nor smooth. Both strain maps of DIC and random fiber model can be able to reflect the influence of the microstructure fibers on the strain concentration around the crack tip of this bio-composite. And the homogeneous finite element model can just give approximately a region of the strain concentration.

\section{Conclusions}

The comparisons between experiments and both numerical results help ones to detail understand the results and phenomenon of DIC experiments; to improve the numerical models in order to be closer to the experimental phenomenon and to increase the knowledge about the fracture behaviour of this bio-composite.

1. We have realized a direct numerical simulation of fracture behaviour for random short spruces fibers reinforced composites. A finite element model with random spruce fibers for PP composite is developed using ABAQUS code and the preprocessed program of the random fiber elements generation in FORTRAN. For calculations, wood fibers have been considered as linear elastic bodies, polypropylene matrix as an elastic-plastic material.

2. The tensile behavior of the composite is simulated by this random fiber finite element model in order to consider the influence of volume content and diameter of fiber on its macroscopic tensile behavior.

3. The influence of natural spruces fibers on fracture behavior of the composite is predicted by this random fiber FE model; meanwhile, its macroscopic fracture parameters are calculated by a homogeneous FE model with the macroscopic tensile stress-strain curve that is predicted by the random fiber FE model.

4. Then, numerical results of both homogeneous and random models have been completely compared with experimental results that have been obtained by digital image correlation method. This comparison indicates that random fiber FE model of random short spruce fibers reinforced composites can be able to fairly reflect the influence of random fibers microstructure in the composite on its fracture behavior. The numerical predictions of the fracture parameters of the composite, such as stress, strain, J-integral, COD and CTOD, etc., are consistent with the DIC experimental results.

5. The calculation of both random fiber and homogeneous FE model and their comparison with experiments show that the average values of J-integral in a region in the front of the crack tip from both numerical FE models are in good agreement with the average J value of DIC experiment in the same region when the numerical and experimental CT specimens of the short spruce fiber reinforced composite are subjected to the same extension at their loading point.

Acknowledgements: The first author is grateful to the Poitou-Charentes Region for its financial support and to LMPM for the invitation, would also like to thank the NSFC(No:10972155) in China. 


\section{References}

1. G. Marsh, Next step for automotive materials. Materialstoday: 36-43. (2003).

2. D. Nabi Saheb, Jog, J.P., Natural fiber polymer composites: A review, ADV POLYM TECH, 18/4: 351-363, (1999).

3. Wu, J., Yu, D., Chan, C.-M., Kim, J. and Mai, Y.-W, "Effect of fiber pretreatment condition on the interfacial strength and mechanical properties of wood fiber/PP composites", J APPL POLYM SCI, 76: 1000-1010 (2000).

4. S.M. Zebarjad, Bagheri, R., Lazzeri, A. and Serajzadeh, S., Fracture behaviour of isotactic polypropylene under static loading condition, Materials and Design, 24: 105-109 (2003).

5. D.R. Moore, A. Pavan, J.G. Williams, Fracture Mechanics Testing Methods for Polymers Adhesives and Composites, ESIS Publication 28, (Elsevier Science Ltd. , 2001)

6. USDA Forest Service, WOODFIBER-PLASTIC COMPOSITES, Virigin and Recycyled Wood Fiber and Polymers for Composites, May 1-3, 1995, Madison, Wisconsin, Sponsored by the USDA Forest Service in cooperation with the University of Toronto the University of Wisconsin and the Forest Products Society.

7. S.M. Zebarjad, Bagheri, R., Lazzeri, A. and Serajzadeh, S., Fracture behaviour of isotactic polypropylene under static loading condition, Materials and Design, 24: 105-109,(2003).

8. M.G.D. Geers, R. de Borst, T. Peijs. Mixed numerical-experimental identification of non-local characteristics of random-fibre-reinforced composites. Composites Science and Technology, 59: 1569-1578, (1999).

9. J.A. Cordes, A.T. Chang and J.L. Charvet. Predicting fracture from the tensile properties of a composite biomaterial. Composites Part A 29A: 1475-1480, (1998).

10. AS Kobayashi, editor. Handbook on experimental mechanics. Revised Ed. New York: Wiley; 1993.

11. WH Peter, Ranson WF. Digital imaging technique in experimental mechanics. Opt. Eng; 21(34): 427-31, (1982).

12. MA Sutton, The effects of subpixel image restoration on digital correlation error estimates. Opt . Eng; 27(3): 173-5(1988).

13. J. Brillaud, Lagattu, F., Limits and possibilities of laser speckle and white -light imagecorrelation methods: theory and experiments. APPL OPTICS, 41/31: 6603-6613, (2002).

14. T.Q. Lam. Mechanical characterization of recycled polymer composites reinforced by natural fibers. Identification of fracture mechanisms by digital image correlation technique. $\mathrm{PhD}$. Thesis, LMPM ENSMA, (2004).

15. T.Q. Lam, F. Lagattu, J. Brillaud and J. Barbier, Influence of natural fibre reinforcement on microstructural and mechanical properties of polypropylene. ECCM11 congress in Greece, May, (2004). 